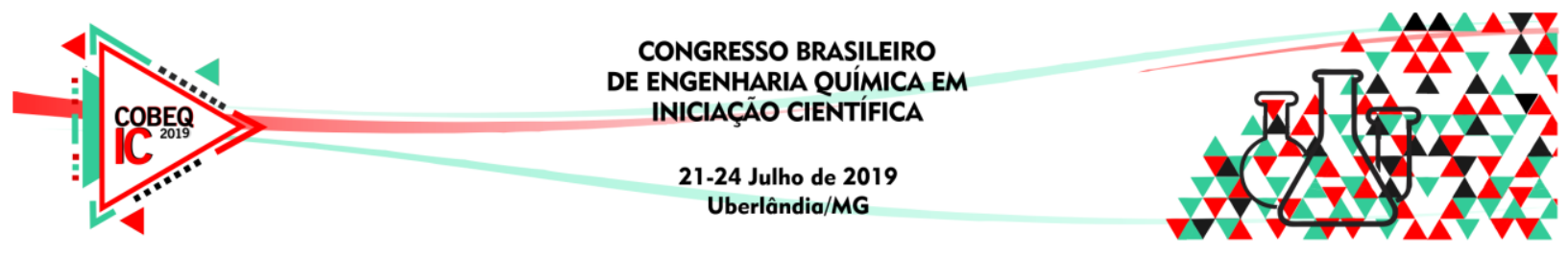

\title{
SÍNTESE DA ZEÓLITA ZSM-22 A PARTIR DO MÉTODO DO GEL SECO TIPO SAC
}

\author{
L. P. G. VASCONCELOS ${ }^{1}$, H. M. H. DE SOUZA ${ }^{1}$, J. R. SANTOS $^{1}$, M. M. URBINA ${ }^{1}$ e D. P. \\ S. SILVA ${ }^{1}$ \\ ${ }^{1}$ Universidade Federal de Alagoas, Centro de Tecnologia \\ E-mail para contato: lary_plentz@hotmail.com
}

\begin{abstract}
RESUMO - Neste trabalho foi proposto o estudo da síntese da ZSM-22 a partir do método do gel seco tipo conversão assistida de vapor (SAC). Foram variados alguns parâmetros de síntese como a quantidade de direcionador ( $\left.\mathrm{HDMA} / \mathrm{SiO}_{2}\right)$, a basicidade $\left(\mathrm{OH}^{-} / \mathrm{SiO}_{2}\right)$ e o tempo de cristalização. As amostras obtidas foram caracterizadas inicialmente a partir da análise de DRX, e as amostras que apresentaram melhores valores de cristalinidade foram então submetidas a análise de TG-DTG. A partir dos resultados obtidos observou-se que com o aumento do tempo de cristalização os picos das amostras estavam melhor definidos até o tempo de 48 horas, tempo este em que a estrutura estava totalmente formada. Após essa condição, a amostra apresentou picos de estruturas secundárias. Foi observado também que a diminuição da basicidade favoreceu a formação da estrutura pura da zeólita ZSM-22.
\end{abstract}

\section{INTRODUÇÃO}

As zeólitas fazem parte de um grupo de sólidos porosos chamados peneiras moleculares, elas possuem a capacidade de adsorver moléculas compatíveis com as dimensões de seus poros, enquanto impedem a entrada de moléculas incompatíveis (Luna, 2001).

Segundo a IZA (International Zeolite Association) existem registradas atualmente 232 estruturas zeolíticas, dentre essas temos um pouco mais de 40 que são encontradas na natureza e as demais são obtidas através de sínteses (Baerlocher; 2007). Dentre essas estruturas está a zeólita sintética ZSM-22, sintetizada pela primeira vez em meados dos anos 80 (Mobil, 1990). Este material apresenta uma estrutura tipo TON, que abrange as zeólitas ZSM-22, Theta-1, Nu-10, KZ-2 e ISI-1 que são ricas em silício. A ZSM-22 é constituída por anéis de 5, 6, e 10 membros, apresentando sistema de canais unidimensionais, não comunicantes, com canais livres em forma de elipse que apresentam diâmetro de 0,45 a 0,55 $\mathrm{nm}$.

As indústrias petroquímicas têm a ZSM-22 como bastante promissora na área de refino de petróleo, em processos de isomerização, craqueamento de metanol e nafta para as reações de olefinas leves como catalisador seletivo de formas, tendo em vista que seu desempenho está ligado diretamente ao tamanho e morfologia de seus cristais (Jamil et al., 2014). 


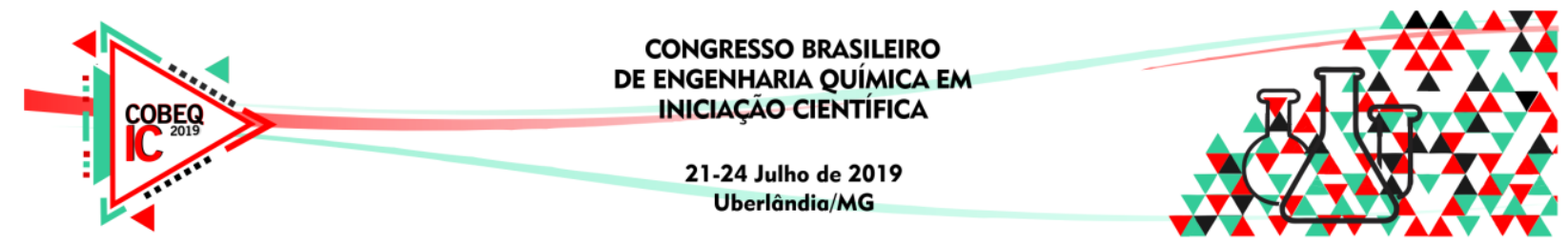

Devido a este grande interesse industrial nesta zeólita, este trabalho foi direcionado na síntese da ZSM-22 a partir do método do gel seco tipo SAC, com a variação de parâmetros de síntese (composição do gel e tempo de cristalização), com o objetivo de verificar a influência dos mesmos na obtenção da estrutura. As amostras obtidas foram caracterizadas por difratometria de raios-X (DRX). Com os resultados de cristalinidade as melhores amostras (mais cristalinas e puras) foram caracterizadas por análises termogravimétricas (TG-DTG).

\section{METODOLOGIA}

Para as sínteses das amostras da ZSM-22, foram necessários os seguintes reagentes: sulfato de alumínio (Merck, 51-59\%), sílica coloidal AS-40 (Aldrich, 40\%), hidróxido de potássio (Sigma-Aldrich), diaminohexano (Aldrich, 99\%), água destilada e sementes de cristalização, sintetizadas através do método hidrotérmico, em reator Parr de 1L (modelo 4848 Reactor Controller) a $160^{\circ} \mathrm{C}$, utilizando a seguinte composição do gel: 27 HDMA : $12 \mathrm{~K}_{2} \mathrm{O}$ : $1,00 \mathrm{Al}_{2} \mathrm{O}_{3}: 90 \mathrm{SiO}_{2}: 3600 \mathrm{H}_{2} \mathrm{O}$.

\subsection{Síntese do Gel Seco}

As amostras de ZSM-22 foram sintetizadas modificando as composições do gel seco onde houve variação na razão de $\mathrm{HDMA} / \mathrm{SiO}_{2}(0,15,0,3$ e 0,6$)$ e na razão de $\mathrm{OH}^{-} / \mathrm{SiO}_{2}(0,1$ e $0,2)$ do gel mantendo a temperatura constante a $140{ }^{\circ} \mathrm{C}$, gerando um total de 6 sínteses, conforme mostra a Tabela 1.

Tabela 1 - Composição dos géis de síntese das amostras da ZSM-22

\begin{tabular}{|c|c|c|c|c|c|c|}
\hline \multirow{2}{*}{$\begin{array}{c}\text { Código da } \\
\text { síntese }\end{array}$} & \multicolumn{6}{|c|}{ Composição do gel } \\
\cline { 2 - 7 } & $\mathrm{SiO}_{2} / \mathrm{Al}_{2} \mathrm{O}_{3}$ & $\mathrm{OH}^{-} / \mathrm{SiO}_{2}$ & $\mathrm{HDMA} / \mathrm{SiO}_{2}$ & $\mathrm{H}_{2} \mathrm{O} / \mathrm{SiO}_{2}$ & Temp. $\left({ }^{\circ} \mathrm{C}\right)$ & $\begin{array}{c}\text { Sementes } \\
(\%)\end{array}$ \\
\hline $\mathrm{H} 1$ & 90 & 0,1 & 0,15 & 40 & 140 & 10 \\
\hline $\mathrm{H} 2$ & 90 & 0,1 & 0,3 & 40 & 140 & 10 \\
\hline $\mathrm{H} 3$ & 90 & 0,1 & 0,6 & 40 & 140 & 10 \\
\hline $\mathrm{H} 4$ & 90 & 0,2 & 0,15 & 40 & 140 & 10 \\
\hline $\mathrm{H} 5$ & 90 & 0,2 & 0,3 & 40 & 140 & 10 \\
\hline $\mathrm{H} 6$ & 90 & 0,2 & 0,6 & 40 & 140 & 10 \\
\hline
\end{tabular}

Para iniciar o procedimento de preparação do gel seco, foi necessário o preparo de cinco soluções a partir da dissolução dos reagentes em água destilada: solução A: o hidróxido de potássio solubilizado em $20 \%$ da quantidade total de água; solução B: o direcionador HDMA (1,6 diaminoexano) solubilizado em $30 \%$ da quantidade total de água; solução C: o sulfato de alumínio solubilizado em $20 \%$ da quantidade total de água; solução D: a sílica sol solubilizada em $10 \%$ da quantidade total de água; solução E: a semente dissolvida em $20 \%$ da quantidade total de água.

As soluções foram adicionadas a um béquer que permaneceu em agitação através de um agitador mecânico até a sua homogeneização, esse processo durou em torno de 70 minutos. Terminada esta etapa, o gel formado passou por um processo de secagem em uma estufa a uma temperatura de $60^{\circ} \mathrm{C}$. Por fim, o gel foi moído através de um moinho modelo IKA A11 basic. 


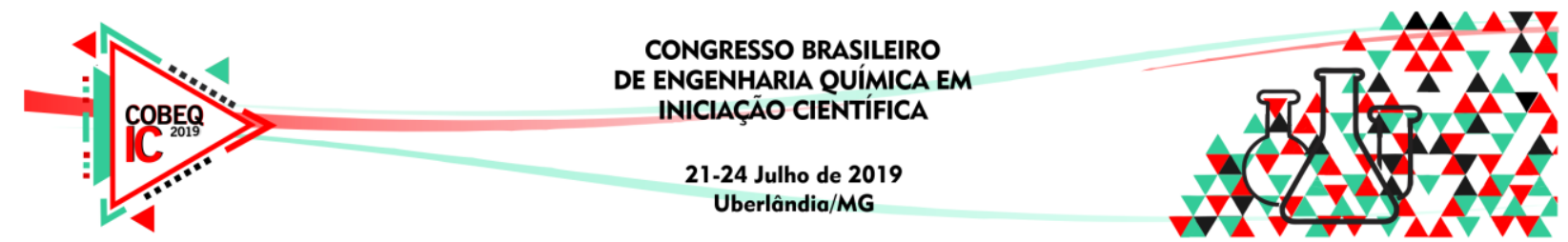

\subsection{Síntese das Amostras da ZSM-22}

Após o término do processo descrito acima, foi colocado em copo de teflon pequeno o gel seco, e em um copo de teflon maior somente água destilada, onde o copo de teflon menor foi inserido a partir de um suporte dentro do copo de teflon maior, que foi fechado e inserido em uma autoclave. Estas autoclaves foram submetidas a uma temperatura de $140{ }^{\circ} \mathrm{C}$ a partir de uma estufa e foram retirados nos devidos tempos de cristalização (6 à 48 horas). Em seguida, as amostras foram lavadas a partir de filtração à vácuo até atingirem um $\mathrm{pH}$ próximo à 7 e foram por fim colocadas para secar à $100^{\circ} \mathrm{C}$ por 24 horas.

\subsection{Caracterização das Amostras}

As amostras foram inicialmente caracterizadas a partir das análises de DRX. Os difratogramas obtidos foram analisados em comparação com o difratograma padrão encontrado no banco de dados da IZA, que se encontra na Figura 1.

Figura 1 - Difratograma padrão da ZSM-22

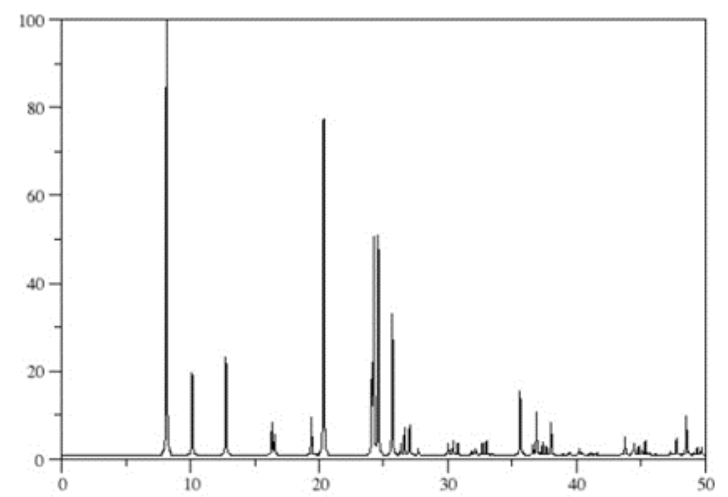

Os principais picos analisados para a formação da estrutura da ZSM-22 estão compreendidos aproximadamente nas regiões $2 \Theta: 18,5-19,5^{\circ}, 20-21^{\circ}, 23-25^{\circ}$ e $25,1-26,1^{\circ}$. A partir do cálculo da área destes picos foi possível então realizar os cálculos da cristalinidade das amostras, calculada através da equação 1 :

$C(\%)=\frac{\sum \text { áreas (amostra) } * 100}{\text { Eáreas (padrão) }}$

Para determinação da cristalinidade das amostras foi considerada como padrão o material sintetizado com maior área para os picos selecionados.

As amostras que apresentaram maiores valores de cristalinidade foram submetidas à análise de TG-DTG, a partir de um equipamento composto por uma termobalança modelo DTG 60 da SHIMADZU, com taxa de aquecimento de $10^{\circ} \mathrm{C} / \mathrm{min}$, na faixa de temperatura ambiente a $800{ }^{\circ} \mathrm{C}$ utilizando atmosfera dinâmica de ar sintético como gás de arraste com fluxo de $50 \mathrm{~mL} / \mathrm{min}$. 


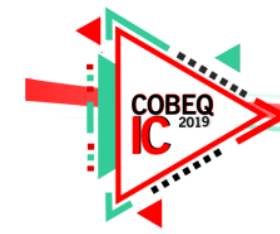

\section{RESULTADOS E DISCUSSÕES}

Na Figura 2 encontram-se os difratogramas comparativos das sínteses realizadas. Podese observar que a partir de 12 horas de cristalização as amostras começam a apresentar os picos característicos da ZSM-22, porém com uma predominância de material amorfo. Com o passar do tempo, esses picos foram aumentando de intensidade e também apresentam uma forma mais definida, enquanto que a parte amorfa foi diminuindo. Para as sínteses realizadas com a basicidade de 0,1 (H1, H2 e H3), a amostra já está totalmente cristalizada a partir de 30 horas, enquanto que para as amostras sintetizadas com a basicidade de 0,2 (H4, H5 e H6) isso só ocorreu com 36 horas de cristalização. Pode-se observar ainda que o aumento no teor de direcionador orgânico utilizado gerou uma diminuição da intensidade dos picos característicos da estrutura da ZSM-22 para ambas as basicidades.

Figura 2 - Difratogramas comparativos das amostras da ZSM-22
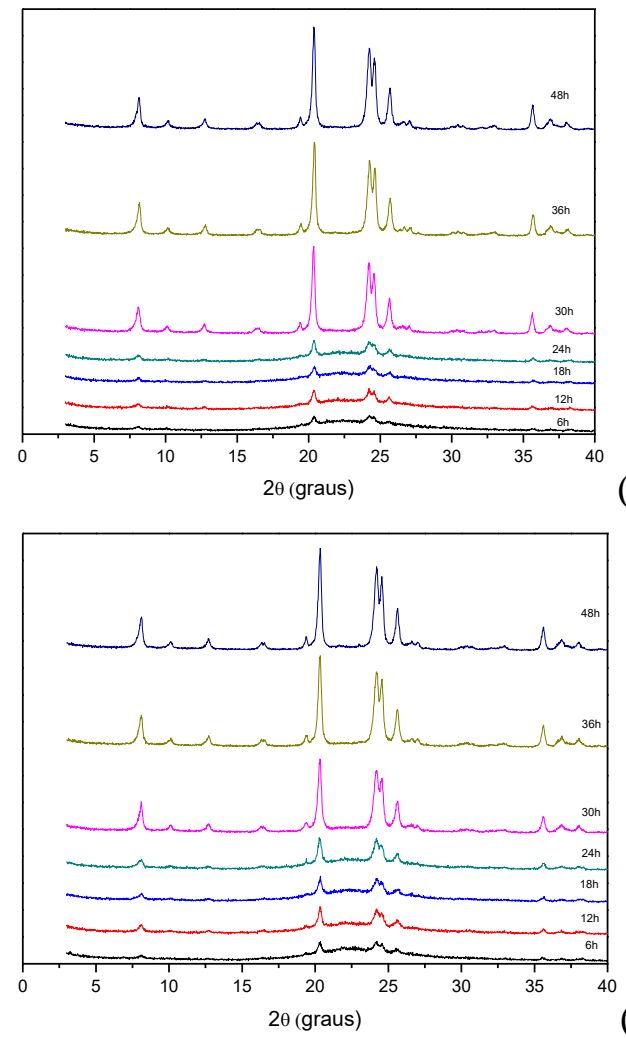

(H3)
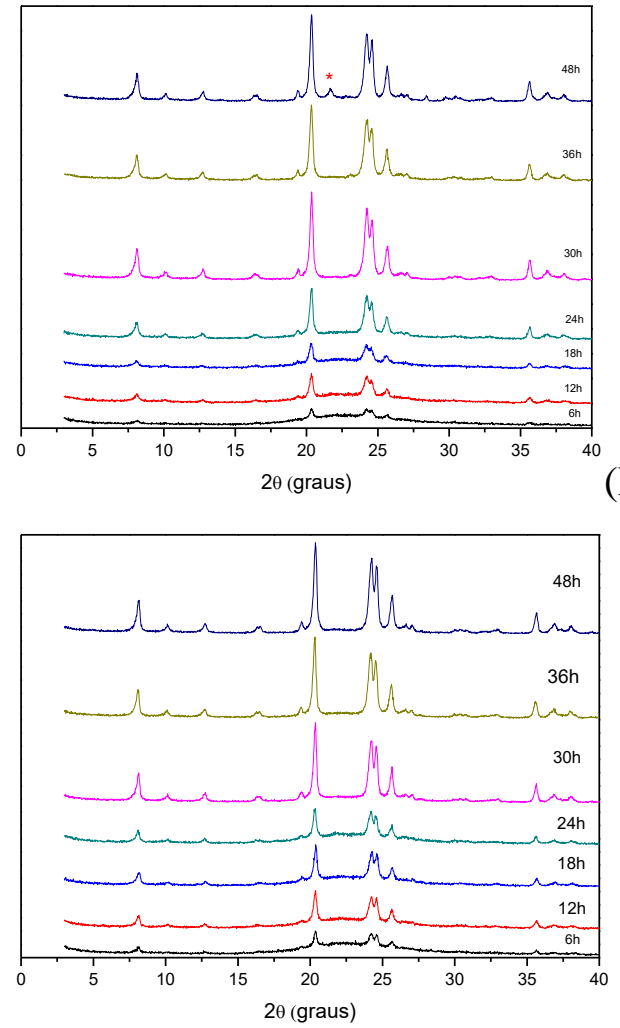

(H4) 

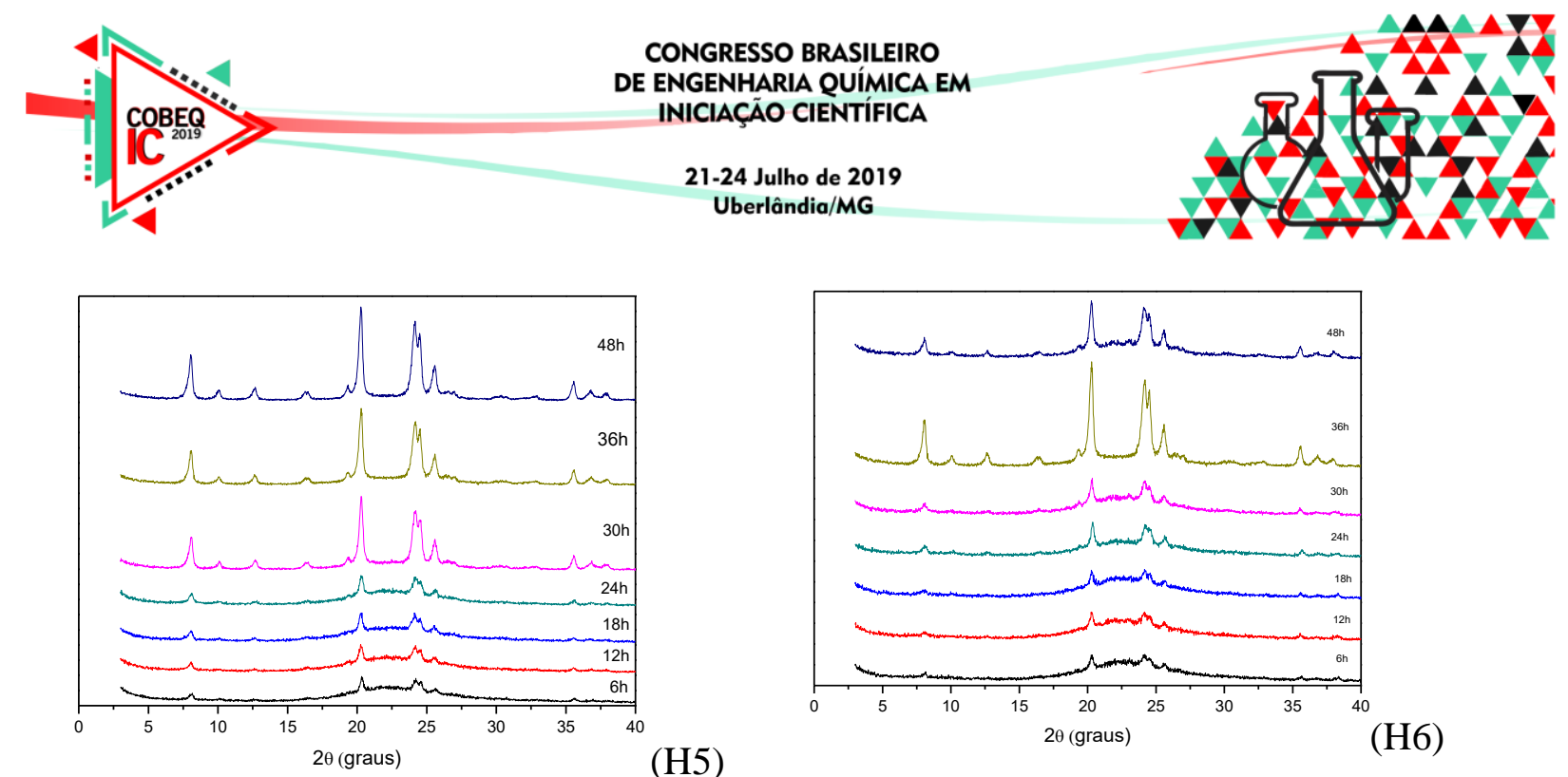

A partir dos difratogramas expostos foi possível calcular a cristalinidade de cada amostra utilizando a equação (1) anteriormente apresentada. A Tabela 2 apresenta o resultado da cristalinidade da melhor amostra de cada síntese, onde estas amostras foram selecionadas para serem submetidas à análise termogravimétrica. A amostra que apresentou o maior somatório das áreas do pico e não apresentou picos de estruturas contaminantes, foi a amostra da síntese $\mathrm{H} 1$ com 48 horas de cristalização, com isso esta foi considerada a amostra padrão, ou seja, ela apresenta cristalinidade $100 \%$. Foi possível observar através dos cálculos de cristalinidade que a amostras com menor basicidade apresentaram melhores resultados quanto à cristalinidade.

Tabela 2 - Cristalinidade das melhoras amostras de cada síntese

\begin{tabular}{|c|c|c|c|c|c|c|}
\hline Síntese & $\mathrm{H} 1$ & $\mathrm{H} 2$ & $\mathrm{H} 3$ & $\mathrm{H} 4$ & $\mathrm{H} 5$ & $\mathrm{H} 6$ \\
\hline \hline $\begin{array}{c}\text { Tempo de } \\
\text { cristalização } \\
\text { (horas) }\end{array}$ & 48 & 30 & 48 & 48 & 48 & 36 \\
\hline $\begin{array}{c}\text { Cristalinidade } \\
(\%)\end{array}$ & 100 & 93 & 99 & 94 & 90 & 64 \\
\hline
\end{tabular}

Na Figura 3 (a) encontram-se as curvas de TG-DTG da amostra H1 com 48 horas, que foi a amostra que apresentou a maior cristalinidade. Todas as outras amostras apresentaram curvas de DTG que mostraram os mesmos eventos de perda de massa. Sendo o evento I atribuído à dessorção de água que estava fracamente ligada à superfície da ZSM-22; o evento II é referente à dessorção do direcionador orgânico que se encontrava fracamente ligado à superfície da ZSM-22 e também à dessorção do direcionador orgânico que se encontrava fortemente ligado à superfície da ZSM-22. Já o evento III refere-se à perda de água estrutural por condensação dos grupos silanol e à remoção de quaisquer fragmentos formados a partir do modelo orgânico (SOUZA et al.; 2017). 


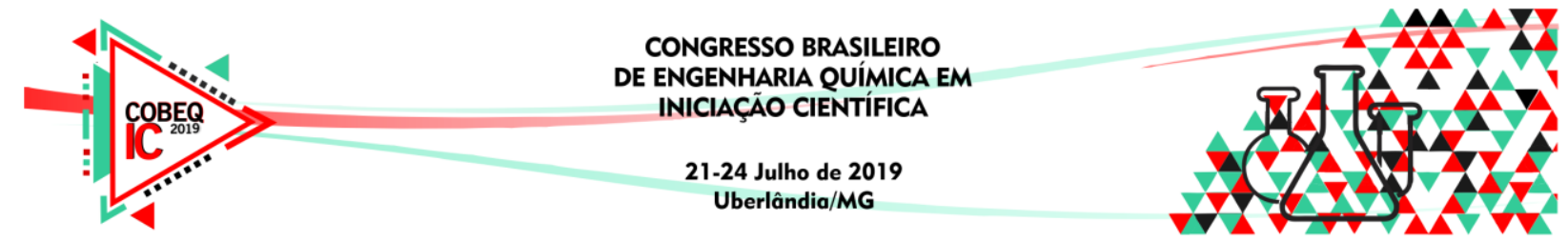

Figura 3 - (a) TG-DTG da amostra H1 com 48 horas; (b) curvas de TG de todas as amostras selecionadas.

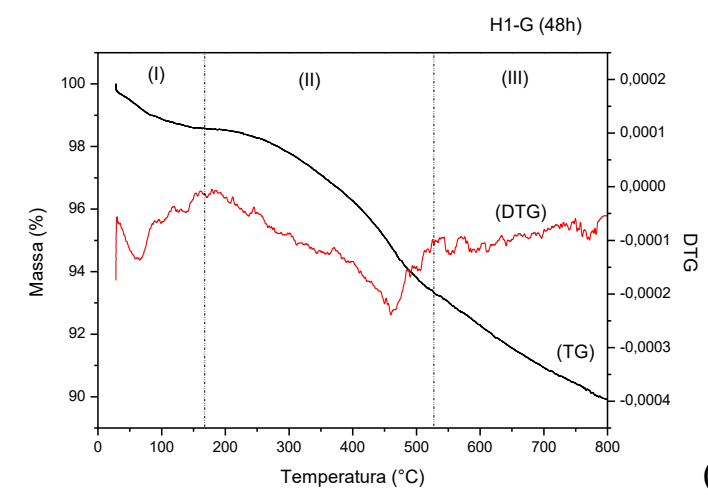

(a)

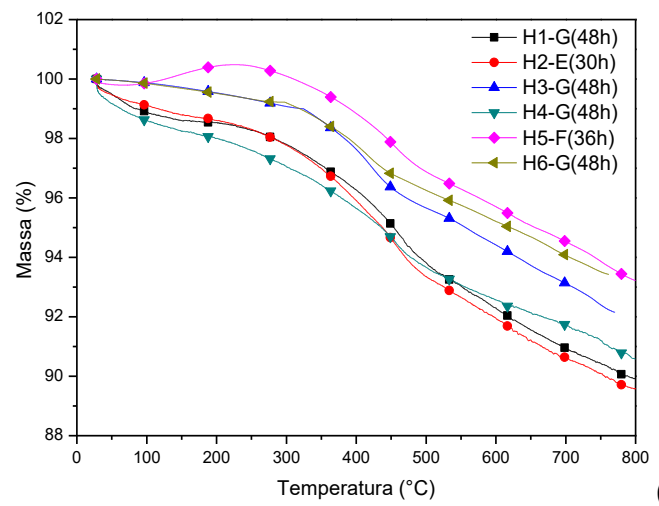

(b)

A partir da Figura 3 (b) pode-se observar as curvas de TG, que representam a perda de massa em porcentagem das amostras com o aumento gradual da temperatura. A partir destas curvas foi construída a Tabela 3 , que mostra a perda de massa de cada amostra com o aumento da temperatura até $800{ }^{\circ} \mathrm{C}$.

Tabela 3 - Perda de masssa (\%) das amostras selecionadas

\begin{tabular}{|c|c|c|c|c|c|c|}
\hline Síntese & $\mathrm{H} 1$ & $\mathrm{H} 2$ & $\mathrm{H} 3$ & $\mathrm{H} 4$ & $\mathrm{H} 5$ & $\mathrm{H} 6$ \\
\hline \hline $\begin{array}{c}\text { Tempo de } \\
\text { cristalização } \\
\text { (horas) }\end{array}$ & 48 & 30 & 48 & 48 & 48 & 36 \\
\hline $\begin{array}{c}\text { Perda de } \\
\text { massa (\%) }\end{array}$ & 10 & 10,35 & 7,55 & 9,25 & 8,96 & 6,99 \\
\hline
\end{tabular}

Ao comparar as amostras das sínteses correspondentes (H1 e H4, H2 e H5, H3 e H6), pode-se observar que as amostras sintetizadas com uma maior basicidade apresentaram uma menor perda de massa que as outras. Esse fato pode ser devido a que nas amostras que apresentaram uma maior concentração de íons $\mathrm{K}^{+}$houve uma menor incorporação de direcionador orgânico nos poros das amostras, causando assim uma menor perda de massa em relação à dessorção do direcionador orgânico.

\section{CONCLUSÕES}

A partir deste trabalho pode-se concluir que o aumento da basicidade na composição do gel precursor da zeólita ZSM-22 causou um retardamento na formação na estrutura da ZSM22 na sua forma cristalina bem como uma menor incorporação do direcionador orgânico nos poros do material, o que pode formar amostras com diâmetros de poro menor que as sintetizadas com uma basicidade menor. Concluiu-se também que o aumento no teor de direcionador orgânico também gerou uma diminuição na intensidade dos picos das amostras.

\section{REFERÊNCIAS}

BAERLOCHER, C.; MEIER, W.M.; OLSON, D.H.; Atlas of zeolite framework types, 5th ed. Amsterdam: Elsevier, 2007. 
JAMIL, A. K.; MURAZA, O.; SANHOOB, M.; TAGO, T.; KONNO, H.; NAKASAKA, Y.; MASUDA, T. Controlling naphtha cracking using nanosized TON zeolite synthesized in the presence of polyoxyethylene surfactant. Journal of Analytical and Applied Pyrolysis, v. 110, n. 1, p. 338-345, 2014.

LUNA, F. J.,SCHUCHARDT, U. Modificação de Zeólitas para uso em Catálise; Química Nova;v. 24, p. 885-892; 2001.

MOBIL. Mobil oil Corporation (New York, N. Y.). Ernest W. Valyocsik. SYNTHESIS OF ZEOLITE ZSM-22.New York. Estados Unidos patente US 4902 406, 1984 jul 10, 20 fevereiro 1990.

SOUSA, L. V., SILVA, A. O. S., SILVA, B. J. B., TEIXEIRA, C. M., ARCANJO, A. P., FRETY, R., PACHECO, J. G. A. Fast synthesis of ZSM-22 zeolite by the seed-assisted method of crystallization with methanol; Microporous and Mesoporous Materials; v. 254; p. 1-9; 2017. 